\title{
Compact configuration ultra-wideband microwave filter using quarter-wave length short-circuited stub
}

\begin{abstract}
In this paper, a compact 5 poles ultra-wideband (UWB) microwave filter is designed by using a quarter wave short-circuited stubs. The UWB filter bandwidth is covered from $2.75 \mathrm{GHz}$ to 10.0 GHz. This filter designed is developed from a low pass Chebychev prototype with a 0.1 $\mathrm{dB}$ passband ripple. The first pattern layout uses 5 vias connecting to the ground. This pattern layout is then re-arranged to reduce the total area space needed on microstrip by maintaining the optimum filter response. Finally, a compact microwave filter with $21 \mathrm{~mm}$ times $16.1 \mathrm{~mm}$ dimension is presented here with excellent scattering characteristic results.
\end{abstract}

Keyword: Component; Formatting; Insert; Style; Styling 